\section{Queda entre idosos no Brasil e sua relação com o uso de medicamentos: revisão sistemática}

\author{
Falls in elderly Brazilians and the relationship to \\ medication: a systematic review
}

\author{
1 Escola de Farmácia, \\ Universidade Federal de Ouro \\ Preto, Ouro Preto, Brasil. \\ Correspondência \\ C. P. Rezende \\ Escola de Farmácia, \\ Universidade Federal de \\ Ouro Preto. \\ Campus Universitário Morro \\ do Cruzeiro, Bairro Bauxita, \\ Ouro Preto, $M G$ \\ 35400-000, Brasil. \\ cris7paula@gmail.com
}

\begin{abstract}
Falls in the elderly, often classified as accidental, are frequently related to medication, generally involving poor prognosis and thus becoming a public health issue. The purpose of this systematic review was to identify published Brazilian studies on medication as a risk factor for falls or fall-related fractures in the elderly. The search covered the LILACS, PubMed, and SciELO indexes using the descriptors falls, elderly, and pharmaceutical preparations/medications/medicines/ drugs or specific drug classes. A total of 340 articles presented data on prevalence, incidence, and risk factors associated with medication and falls or fall-related fractures, but only 6 pharmacoepidemiological studies were examined because they were conducted specifically in Brazilian samples. The main drug classes associated with increased risk of falls: antidepressants, sedatives, anxiolytics, and diuretics. The promotion of rational drug use in geriatrics requires well-designed studies to produce robust scientific data.
\end{abstract}

Accidental Falls; Aged; Drug Utilization
Cristiane de Paula Rezende 1 Maria Ruth Gonçalves Gaede-Carrillo 1 Elza Conceição de Oliveira Sebastião ${ }^{1}$

\section{Introdução}

Nas últimas décadas, observou-se um nítido envelhecimento populacional em todo o mundo, e especialmente no Brasil houve uma diminuição da taxa de fecundidade paralelamente ao aumento da longevidade 1,2. De acordo com a Organização Mundial da Saúde (OMS), o idoso é o indivíduo que apresenta idade igual ou acima de 60 anos (quando vivem em países em desenvolvimento, caso do Brasil) e 65 anos ou mais em países desenvolvidos 3,4. Segundo dados do Instituto Brasileiro de Geografia e Estatística (IBGE. http:/ / www.ibge.gov.br, acessado em 05/Mar/2010), a população idosa ampliou de 7,3\% em 1991 para $8,6 \%$ em 2000; a estimativa é que atinja $20 \%$ em 2050. Assim sendo, o país está deixando de ser predominantemente jovem e se tornando mais maduro 5 .

Esse aumento da população idosa no Brasil, evidenciado pela mudança da pirâmide demográfica brasileira, trouxe como consequência a mudança no perfil das necessidades sanitárias, uma vez que as doenças que acometem a terceira idade são na maioria das vezes crônico-degenerativas, distúrbios mentais, patologias cardiovasculares, câncer e estresse 6 , cujo tratamento envolve uma gama relativamente grande de medicamentos que podem ser utilizados isoladamente ou em associação ${ }^{2}$. Cada um desses fármacos apresenta um perfil de reações adversas que necessita ser investigado e avaliado para uso 
racional 7 , inclusive para prevenir a ocorrência de doenças iatrogênicas, em especial as quedas que podem ser induzidas por medicamentos 8 . Também é importante a perda do equilíbrio postural, doenças neurológicas e a inadequação ao ambiente a que o idoso está exposto, já que a associação de todos esses fatores está ligada à frequente ocorrência de quedas 9 .

Conceitualmente, as quedas são definidas como um deslocamento não intencional do corpo para um nível inferior à posição inicial com incapacidade de correção em tempo hábil, determinado por circunstâncias multifatoriais comprometendo a estabilidade 10,11,12,13. Este evento, apesar de muitas vezes ser considerado como normal na terceira idade, constitui um problema de saúde pública, pois além das fraturas, de alta prevalência em idosos 13,14, gera outras consequências como, diminuição da qualidade de vida, medo de andar e perda da capacidade de realização de tarefas do dia a dia, sendo uma das principais causas de hospitalização e morte em geriatria 15 . Um estudo realizado nos países da União Europeia demonstrou que as quedas entre idosos constituem a principal causa de morte por motivo dos agravos, porém muitas vezes a causa mortis é registrada mais em função das doenças crônicas e injúria física do que pela própria ocorrência da queda em si 16 .

De acordo com o estudo de delineamento transversal realizado em sete estados brasileiros no ano de 2005 13, a prevalência de quedas em idosos foi de $34,8 \%$, e dentre os que caíram, $55 \%$ relataram a ocorrência de uma única queda no ano anterior à pesquisa. Outro estudo retrospectivo 15 revelou que $37,5 \%$ dos idosos também caíram no último ano e, dentre eles, 29,6\% referiram mais de uma queda. Tais estudos estão de acordo com os dados encontrados em pesquisas realizadas nos Estados Unidos em que cerca de $30 \%$ a $40 \%$ dos idosos residentes em uma comunidade sofrem pelo menos uma queda em sua vida, e essa porcentagem aumenta para $60 \%$ quando os idosos já experimentaram queda no ano anterior 17. A ocorrência das quedas na maioria das vezes é ocasionada pelo somatório de diversos fatores de risco e múltiplas causas, tornando difícil a análise restrita de um fator como causador do episódio 18. No entanto, quedas decorrentes de uma causa extrínseca ao indivíduo são majoritariamente acidentais, sendo única e de difícil repetição; já as quedas recorrentes são derivadas de fatores próprios dos indivíduos, como alterações fisiológicas por conta do envelhecimento, das patologias e do uso de medicamentos 19 .

Há mais de duas décadas estão sendo realizados estudos sobre o uso de medicamentos inapropriados em geriatria $20,21,22,23$, dentre eles estão as pesquisas de Beers et al. 20, Beers 21 e Fick et al. 22, que estabeleceram critérios para definir quais fármacos seriam inadequados para os idosos, pois seus riscos superam os benefícios nessa faixa etária. Contudo, no Brasil, ainda são largamente utilizados medicamentos impróprios na farmacoterapia geriátrica 24,25,26.

A demanda pelo atendimento de melhor qualidade e cuidado em saúde aos pacientes, associada com a necessidade de uso racional de medicamentos, necessita estar calcada em estudos que retratem a realidade brasileira, vale dizer, uma prática baseada em evidências científicas 15,27 . Uma vez que não foi encontrada nenhuma revisão sistemática sobre a farmacoepidemiologia das quedas em idosos no Brasil, verificou-se a necessidade de identificar, analisar e qualificar sistematicamente as publicações existentes sobre esse assunto, que é o objetivo da presente revisão.

\section{Metodologia}

Foi realizada uma revisão sistemática dos estudos publicados sobre a associação do uso de medicamentos com a ocorrência de quedas, sem limites de data de publicação dos estudos, ou seja, envolvendo todo o período disponível pelo conjunto de base de dados utilizados.

\section{Critérios de inclusão e exclusão dos estudos}

Foram incluídos na revisão estudos observacionais analíticos realizados no Brasil, que apresentassem dados estatísticos do evento queda (com ou sem fratura) e que associassem a queda ao uso de medicamentos, sendo eles publicados em português, espanhol ou inglês. Os sujeitos participantes das pesquisas selecionadas eram de ambos os gêneros, que apresentassem 60 anos ou mais de idade, sem limites do tamanho de amostra. Foram excluídos estudos sobre quedas associadas a fatores fisiopatológicos próprios do envelhecimento, bem como aqueles que analisavam as consequências do evento. Além desses, também foram excluídos estudos realizados fora do Brasil e aqueles que não disponibilizaram o resumo ou, quando ele estava presente, era insuficiente para uma análise primária.

\section{Busca bibliográfica}

Foi realizada uma revisão sistemática para identificar estudos que atendessem aos critérios de inclusão, utilizando as bases de dados eletrônicas PubMed (base de dados do MEDLINE), SciELO (Scientific Electronic Library Online) e LILACS 
(Literatura Latino-Americana e do Caribe em Ciências da Saúde). Inicialmente, a pesquisa foi feita apenas pela utilização dos descritores Medical Subject Headings (MeSH). Porém, em razão do pequeno número de artigos encontrados quando se utilizou o termo "accidental falls", optouse também por fazer a busca com uso de outros termos sinônimos aos descritores MeSH que poderiam estar presentes no título e no resumo do estudo. A princípio, a busca foi realizada utilizando três termos: "accidental falls" ou "falls", "elderly" e "medication". O último foi também substituído por "pharmaceutical preparations", "medicines", "drugs" ou por grupos de medicamentos específicos tais como psychotropics, benzodiazepines, anticholinergics, antihypertensives, cholinesterase inhibitors, antiepileptics, diuretics, beta-blockers, sedatives hypnotics, neuroleptics, antipsychotics, narcotics e nonsteroidal anti-inflammatory drugs. Foram utilizadas ferramentas de refinamento quando elas estavam disponíveis, como nas bases MEDLINE e LILACS. Foram selecionados somente os estudos desenvolvidos com população geriátrica.

\section{Procedimento da revisão}

Todos os resumos dos artigos identificados na busca foram avaliados observando se preenchiam ou não os critérios de inclusão. Aqueles selecionados foram analisados integralmente por dois investigadores de modo independente. Quando houve discordâncias, elas eram discutidas e se buscava um consenso. Os estudos incluídos foram avaliados segundo as variáveis estudadas: tipo de estudo epidemiológico; temporalidade da coleta de dados nos estudos; o ano de publicação; média de idade dos sujeitos da pesquisa; tamanho amostral; definição específica de quedas; frequência do evento; fatores de risco analisados; associação farmacoepidemiológica entre o uso de medicamentos e quedas. Também foram listados os grupos de medicamentos ou os próprios medicamentos responsáveis pelas quedas.

O sistema usado no presente trabalho como forma de agrupar as diferentes classes medicamentosas foi a classificação ATC - Anatomical Therapeutic Chemical Code, conhecida internacionalmente e adotada pela OMS, que consiste em classificar os fármacos em diferentes grupos e subgrupos (níveis), de acordo com o órgão ou sistema sobre o qual atuam e, posteriormente, segundo as suas propriedades terapêuticas, farmacológicas e químicas podendo os subgrupos chegar ao quinto nível ${ }^{28}$. As medidas de associação estatística entre o uso de medicamentos e as quedas foram apresentadas conforme o referido nos artigos.

\section{Resultados}

As buscas eletrônicas foram realizadas no período de março a junho de 2010 e resultaram em um total de 340 artigos. O organograma que ilustra a metodologia de exclusão/inclusão de artigos está apresentado na Figura 1. Dentre os estudos selecionados, $53(15,6 \%)$ relacionaram as quedas com um ou mais tipo de medicamentos alvo. No entanto, 47 foram realizados fora do Brasil, o que levou a exclusão deles à análise dos dados estatísticos das quedas (com ou sem fratura) e à associação do evento ao uso de medicamentos. Ao fim, restaram seis artigos realizados com a população brasileira que foram objetos de estudo desta revisão sistemática.

A sistematização dos resultados dos artigos incluídos na revisão sistemática foi apresentada nas Tabelas 1 e 2, que apresentam as variáveis estudadas.

\section{Descrição dos estudos incluídos}

A Tabela 1 demonstra de forma agrupada as características dos estudos: data, desenho epidemiológico do estudo (tipo), evento, porcentagem de mulheres incluídas, média de idade, existência de definição específica de quedas, sua frequência em cada estudo e quais os fatores de risco analisados. Os resultados dessa tabela serão detalhados abaixo.

Nota-se que os artigos são relativamente recentes, sendo o mais antigo publicado no ano de 2000.

Os estudos selecionados foram todos retrospectivos, classificados como epidemiológicos analíticos observacionais transversais $13,29,30$ ou caso-controle 31,32,33. As investigações de delineamento transversal 12,30 elegeram como sujeitos participantes de um grupo de atividades físicas vinculados à universidade ou idosos que viviam na comunidade 29 . Todos os casos dos estudos caso-controle foram hospitalizados por conta da ocorrência de fraturas decorrentes de quedas e apresentaram como controle os pacientes hospitalizados por outras causas, pareados pela idade 31,32 e pelo gênero ${ }^{31}$. Na outra pesquisa $\mathbf{3 3}$, os controles foram pareados pela idade e gênero de pessoas que viviam na comunidade na mesma região dos casos.

O número total de participantes de todos os estudos corresponde a 1.449 idosos, e mais de $70 \%$ dos sujeitos estudados são mulheres. Quando o evento estudado foi queda, Chaimowicz et al. 29 encontraram que, dentre os $161,58,4 \%$ eram mulheres, embora neste estudo não tenha sido estratificada a ocorrência do evento pelo gênero. Quando o evento foi fratura decorrente de 
Figura 1

Diagrama da análise dos estudos e processo de seleção.

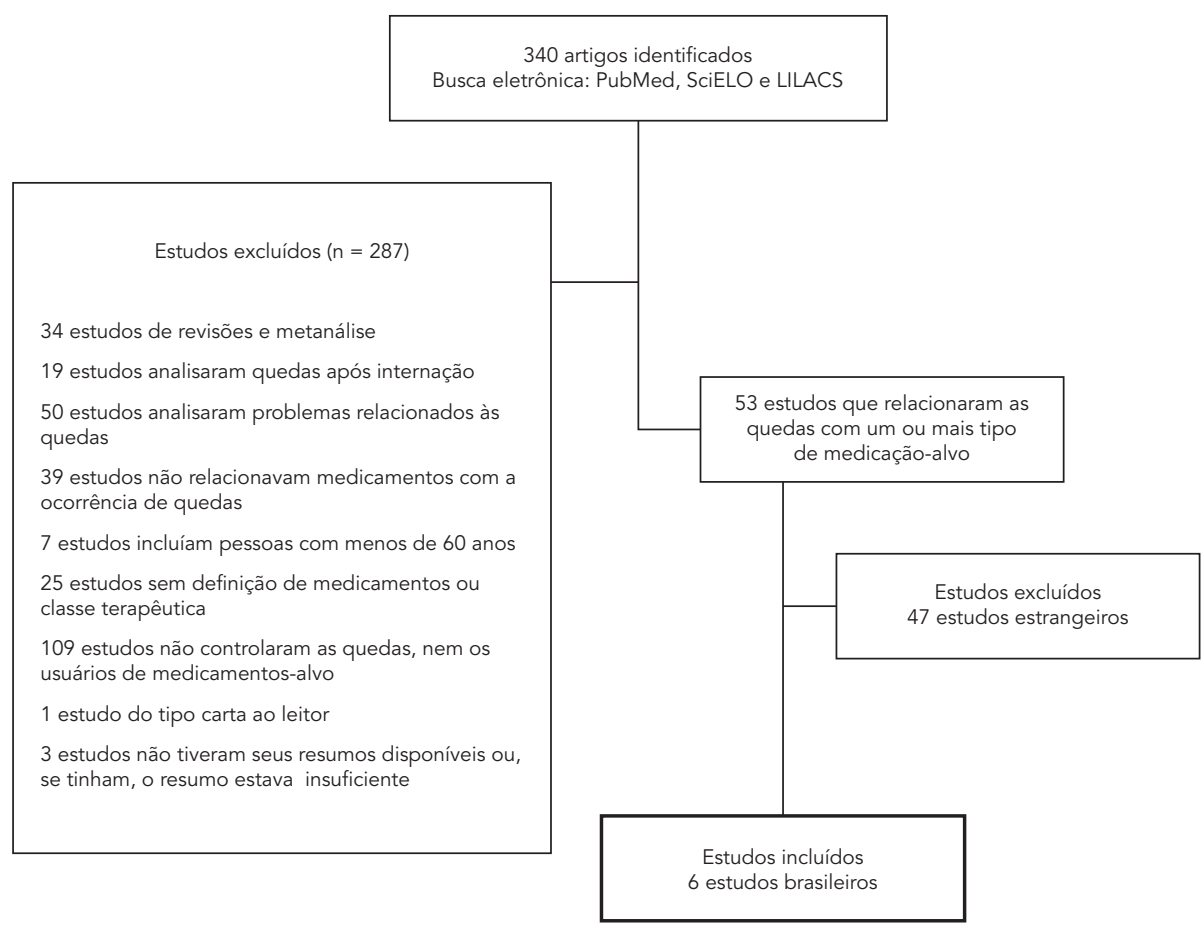

Tabela 1

Características dos seis estudos selecionados para a revisão sistemática.

\begin{tabular}{|c|c|c|c|c|c|c|c|}
\hline Referência & $\begin{array}{c}\text { Ano de } \\
\text { publicação }\end{array}$ & Data do estudo & $\begin{array}{l}\text { Tipo de } \\
\text { estudo }\end{array}$ & $\begin{array}{c}\text { Número de } \\
\text { pessoas/ } \\
\text { Mulheres (\%) }\end{array}$ & $\begin{array}{l}\text { Média de } \\
\text { idade (anos) }\end{array}$ & Evento & Frequência do evento \\
\hline $\begin{array}{l}\text { Chaimowicz } \\
\text { et al. } 29\end{array}$ & 2000 & Agosto/Dezembro 1998 & Transversal & $\begin{array}{c}161 \text { idosos; } 58,4 \% \\
\text { mulheres }\end{array}$ & 71 & Queda & $17 \%$ relataram queda \\
\hline $\begin{array}{l}\text { Coutinho \& } \\
\text { Silva } 31\end{array}$ & 2002 & 1998-1999 & Caso-controle & $\begin{array}{c}169 \text { casos; } 77,5 \% \\
\text { mulheres }\end{array}$ & $\begin{array}{c}70,7 \text { casos; } \\
71,9 \\
\text { controles }\end{array}$ & $\begin{array}{c}\text { Fratura } \\
\text { decorrente } \\
\text { de queda }\end{array}$ & $\begin{array}{l}100 \% \text { deles, internados } \\
\text { vítimas de fratura }\end{array}$ \\
\hline $\begin{array}{l}\text { Rozenfeld } \\
\text { et al. } 12\end{array}$ & 2003 & Julho/Dezembro 1996 & Transversal & $\begin{array}{c}634 \text { idosas; } \\
\text { 100\% mulheres }\end{array}$ & $\begin{array}{c}69,3 ; 61,4 \% \\
(60-69)\end{array}$ & Queda & $37,4 \%$ relataram queda \\
\hline $\begin{array}{l}\text { Guimarães \& } \\
\text { Farinatti } 30\end{array}$ & 2005 & ND & Transversal & $\begin{array}{c}30 \text { idosas; } 100 \% \\
\text { mulheres }\end{array}$ & $66-86 ; 75 \pm 5$ & Queda & $43 \%$ relataram queda \\
\hline $\begin{array}{l}\text { Hamra et } \\
\text { al. } 32\end{array}$ & 2007 & Janeiro/Dezembro 2004 & Caso-controle & $\begin{array}{c}205 \text { casos; } 71,7 \% \\
\text { mulheres }\end{array}$ & $75,1(60-97)$ & $\begin{array}{c}\text { Fratura } \\
\text { decorrente } \\
\text { de queda }\end{array}$ & $\begin{array}{c}100 \% \text { deles, internados } \\
\text { vítimas de fratura }\end{array}$ \\
\hline $\begin{array}{l}\text { Coutinho } \\
\text { et al. } 33\end{array}$ & 2008 & $2002-2003$ & Caso-controle & $\begin{array}{c}250 \text { casos; } 78 \% \\
\text { mulheres }\end{array}$ & $\begin{array}{c}75,5 \text { casos; } \\
75,3 \\
\text { controles }\end{array}$ & $\begin{array}{c}\text { Fratura } \\
\text { decorrente } \\
\text { de queda }\end{array}$ & $\begin{array}{c}100 \% \text { deles, internados } \\
\text { vítimas de fratura }\end{array}$ \\
\hline
\end{tabular}

ND: não demonstrado. 


\section{Tabela 2}

Uso de medicamentos e risco de quedas.

\begin{tabular}{|c|c|c|c|c|c|}
\hline Referência & $\begin{array}{c}\text { Ano de } \\
\text { publicação }\end{array}$ & Evento & Medidas de associação & $\begin{array}{c}\text { Medicamentos associados ao } \\
\text { evento }(p<0,05)\end{array}$ & $\begin{array}{l}\text { ATC dos medicamentos } \\
\text { associados ao evento }\end{array}$ \\
\hline \multirow{8}{*}{$\begin{array}{l}\text { Chaimowicz } \\
\text { et al. } 29\end{array}$} & 2000 & Queda & Teste de Fisher & Ansiolíticos & N05B \\
\hline & & & Medicamentos psicoativos & Antidepressivo & N06A \\
\hline & & & $(t=2,45 ; p=0,02)$ & & \\
\hline & & & Benzodiazepínicos & & \\
\hline & & & $(t=1,97 ; p=0,05)$ & & \\
\hline & & & Benzodiazepínicos e/ou & & \\
\hline & & & antidepressivos & & \\
\hline & & & $(t=2,23 ; p=0,03)$ & & \\
\hline \multirow{4}{*}{$\begin{array}{l}\text { Coutinho \& } \\
\text { Silva } 31\end{array}$} & 2002 & Fraturas & $\mathrm{OR}=1,96$ & Bloqueadores de canais de cálcio & $\mathrm{C0} 8^{\star \star}$ \\
\hline & & decorrentes & & & \\
\hline & & de quedas & $\mathrm{OR}=2,09$ & Ansiolíticos & N05B \\
\hline & & & $\mathrm{OR}=0,40$ & Diuréticos * & $\mathrm{CO} 3 * \star$ \\
\hline \multirow{3}{*}{$\begin{array}{l}\text { Rozenfeld } \\
\text { et al. } 12\end{array}$} & 2003 & Queda & $\mathrm{OR}=1,57$ & Diuréticos & $\mathrm{CO} 3^{\star *}$ \\
\hline & & & $\mathrm{OR}=2,02$ & $\beta$-bloqueadores & C07A \\
\hline & & & $\mathrm{OR}=4,93$ & Ansiolíticos/sedativos & N05B \\
\hline \multirow{4}{*}{$\begin{array}{l}\text { Guimarães \& } \\
\text { Farinatti } 30\end{array}$} & 2005 & Queda & Frequência relativa e & & \\
\hline & & & absoluta (\%) & & \\
\hline & & & 60 & Diuréticos & $\mathrm{CO} 3 * *$ \\
\hline & & & 78 & Psicoativos & $N * \star$ \\
\hline \multirow[t]{9}{*}{ Hamra et al. 32} & 2007 & Fraturas & $\mathrm{OR}=57,46 \% ; \mathrm{RR}=83,11 \%$ & & \\
\hline & & decorrentes & Número de & & \\
\hline & & de quedas & medicamentos usados & & \\
\hline & & & pelos casos e controle & & \\
\hline & & & $51 ; 30$ & Captopril & CO9A \\
\hline & & & $13 ; 1$ & Clonazepam & N05B \\
\hline & & & $28 ; 14$ & Hidroclorotiazida & C03A \\
\hline & & & $9 ; 1$ & Cinarizina & N07C \\
\hline & & & $3 ; 1$ & Flunarizina & N07C \\
\hline \multirow{3}{*}{$\begin{array}{l}\text { Coutinho et } \\
\text { al. } 33\end{array}$} & 2008 & Fraturas & $\mathrm{OR}=2,22$ & Ansiolíticos & N05B \\
\hline & & decorrentes & & & \\
\hline & & de quedas & $\mathrm{OR}=4,42$ & Relaxantes musculares & M03B \\
\hline
\end{tabular}

ATC: Anatomical Therapeutic Chemical Code; IC95\%: intervalo de 95\% de confiança; OR: odds ratio.

* Fator protetor de fraturas decorrentes de quedas;

** Estudos não citaram quais os medicamentos de forma a poderem ser classificados em níveis mais específicos. 
quedas, a ocorrência em mulheres foi ainda maior: $77,5 \%$ no estudo de Coutinho \& Silva 31 , $78 \%$ no estudo de Coutinho et al. 33 e $71,7 \%$ no estudo de Hamra et al. 32 . Pode ser constatado que nas pesquisas nas quais a porcentagem de mulheres foi menor, a frequência de ocorrência de quedas também foi menor.

Verifica-se na Tabela 1 que a média de idade dos participantes dos estudos que analisaram as fraturas decorrentes de quedas foi ligeiramente maior que daqueles envolvendo quedas. Contudo, apenas 2 dos 6 artigos incluídos na revisão 12,33 definem especificamente tal evento.

No estudo de Chaimowicz et al. 29 , a frequência de quedas foi menor que nos demais artigos. Rozenfeld et al. 12 verificaram que a frequência foi de $37 \%$, lembrando que neste estudo a totalidade dos sujeitos era do gênero feminino. Os artigos que apresentam $100 \%$ da frequência do evento se referem a casos de fratura que decorreram de quedas $31,32,33$.

Dentre os 6 artigos analisados, 4 estudos 12,29,31,32 apresentaram como objetivo principal o uso de medicamentos como fator de risco para quedas ou fraturas decorrentes de quedas; um estudo 33 analisou os fatores associados à saúde, e o outro 30 analisou os fatores extrínsecos e intrínsecos para as quedas.

A Tabela 2 apresenta as medidas de associação utilizadas pelos estudos, quais medicamentos estavam associados ao evento e à classificação ATC. O evento novamente foi demonstrado nessa tabela para facilitar a visualização dos dados apresentados. Nenhum dos estudos se referiu à classificação ATC, mas nesta revisão foi utilizada como forma de padronizar a análise.

O estudo de Chaimowicz et al. 29 avaliou a utilização de medicamentos psicoativos e a ocorrência de quedas nos últimos 12 meses, tendo sido utilizado o teste de Fisher, o qual apresentou relação estatística significativa para todos os psicoativos referidos, e também especificamente para os benzodiazepínicos e/ou antidepressivos, controlando-se as variáveis como idade, sexo, visão e audição.

A pesquisa de Coutinho \& Silva 31 fez uma associação entre o uso de medicamentos e a ocorrência de fraturas decorrentes de quedas por meio de odds ratio (OR); a presença de fator de confusão foi investigada por regressão logística condicional. Neste estudo foi verificado um aumento do risco de fraturas em $96 \%$ dos casos entre os usuários de bloqueadores de canais de cálcio e $109 \%$ entre os idosos que usavam benzodiazepínicos.

Rozenfeld et al. ${ }^{12}$ verificaram a associação entre uso de medicamentos e a ocorrência de quedas pela análise estatística e OR. Quedas recorrentes foram estatisticamente associadas com o uso de $\beta$-bloqueadores e com ansiolíticos/ sedativos, após o ajuste para doenças cardiovasculares. A associação dos ansiolíticos/sedativos com ocorrência de quedas foi estatisticamente significante quando também era relatada a hipotensão postural. A ocorrência de uma queda no último ano foi associada ao uso de diuréticos em mulheres que apresentam também distúrbios músculo-esqueléticos.

Guimarães \& Farinatti 30 fizeram a associação do uso de medicamentos com a ocorrência do evento utilizando estatística descritiva, apresentando as frequências relativas referentes aos sujeitos que caíram no último ano e tomavam psicoativos (78\%) e diuréticos (60\%).

O estudo de Hamra et al. 32 foi do tipo casocontrole, cuja associação entre o uso de medicamentos e a ocorrência de fraturas foi realizada pela análise estatística de OR, que foi apresentada em porcentagem. Esse estudo enfatizou que os pacientes usuários de captopril, clonazepam, hidroclorotiazida, cinarizina e flunarizina e que sofreram fraturas decorrentes de quedas foi maior do que o grupo controle.

Coutinho et al. 33 utilizaram a OR como medida de associação e demonstraram que os benzodiazepínicos e relaxantes musculares estavam associados a um risco aumentado de fraturas decorrentes de quedas.

\section{Discussão}

Todos estudos incluídos nesta revisão sistemática são recentes, isso possivelmente está associado ao aumento da população idosa no Brasil 1,2,6 (IBGE. http://www.ibge.gov.br, acessado em 05/Mar/2010), e também pela maior procura dos idosos por atendimento à saúde 6,7,8,34 e, consequentemente, maior interesse em pesquisas sanitárias na área geriátrica 5,6,7.

Na maioria dos estudos 12,30,31,32, a porcentagem de mulheres entre os sujeitos da pesquisa foi maior que $70 \%$, demonstrando o quanto as quedas ou fraturas decorrentes de queda são fatores preocupantes para este gênero. Isso pode ser devido ao fato inerente de maior fragilidade óssea das mulheres 35,36. Dentre elas, a frequência de fraturas osteoporóticas é maior por causa do tamanho de seus ossos, que são menores do que os masculinos, além da diminuição dos hormônios gonadais com o avançar da idade, levando à perda da massa esquelética feminina mais pronunciada do que nos homens ${ }^{37}$. Em estudo transversal 38 , realizado em 100 municipios brasileiros, com 6.616 idosos, $27,6 \%(n=1.826)$ deles relataram ter sofrido queda no último ano, e $11 \%$ 
tiveram fratura; $59 \%$ desses sujeitos eram mulheres. Noutro estudo brasileiro transversal $13 \mathrm{com}$ 4.003 idosos em 7 estados no Brasil, a prevalência de quedas entre mulheres foi de $40,1 \%$, enquanto entre homens foi de $26,5 \%$, demonstrando que as mulheres são potencialmente mais propensas a sofrerem quedas do que os homens. Também se verificou que os idosos que sofreram fraturas decorrentes de quedas eram mais velhos que aqueles que caíram. Essa diferença pode ter sido em função de a ocorrência de fraturas ser maior quando os idosos apresentam um estado mais debilitado e frágil, o que na maioria das vezes ocorre em idade mais avançada 39 . Um estudo multicêntrico realizado com idosos institucionalizados na Austrália apresentou uma média de 1,74 queda por pessoa-ano entre 602 participantes; desses, 70,9\% eram mulheres 40 .

Os critérios de inclusão dos artigos selecionados para essa revisão variaram de acordo com o tipo de evento analisado. Os participantes incluídos nos estudos deveriam ter idade superior a 60 anos de acordo com o Estatuto do Idoso, porém 2 estudos analisaram os idosos a partir de 65 anos ou mais. Quando a análise tratou das quedas, o único critério de inclusão foi a idade; já nos estudos que analisavam as fraturas decorrentes de quedas, os critérios de inclusão foram idade e internação por motivo de fraturas decorrentes de quedas. As fraturas são uma das mais importantes consequências de quedas nos idosos, sendo a prevalência de $5 \%$ entre os que caem 14 . Prevalência ainda maior foi encontrada em 200713 $(12,1 \%)$. Sendo assim, a análise de fraturas decorrentes de quedas é um parâmetro importante para investigar indiretamente a ocorrência das quedas. Por este motivo, estudos brasileiros que apresentaram este evento foram incluídos nesta revisão.

Grande parte dos estudos não define especificamente o termo queda 29,30,31,32, o que pode dificultar as pesquisas, uma vez que pode obscurecer os seus objetivos, levando a vieses de informações dadas pelos sujeitos. Além disso, a comparação entre os estudos pode ser prejudicada, pois diferentes pesquisas podem apresentar resultados diversos por causa da falta de similaridade da metodologia, bem como da definição específica de quedas 12 .

Guimarães \& Farinatti 30 analisaram os fatores de risco extrínsecos para as quedas. Neste estudo foram analisadas as condições de conservação dos locais onde os idosos caíram, a capacidade visual, doenças associadas, flexibilidade, força, equilíbrio e o uso de medicamentos. Dos sujeitos da pesquisa, $55 \%$ relataram que caíram na rua, $38 \%$ deles referiram que o motivo que levou à queda foram tropeços, mais da metade acredita que caiu por distração e falta de atenção, uma vez que estavam caminhando em locais conhecidos. Os indivíduos que referiram ter comprometimento da visão sofreram mais quedas do que aqueles que consideraram boa a sua visão. Após a ocorrência do evento, $41 \%$ das mulheres necessitaram de algum tipo de atendimento médico.

Chaimowicz et al. 29 encontraram uma frequência de quedas menor e de fraturas maior do que os outros estudos transversais 12,30. Essa redução pode ser em função da natureza do estudo porque a ocorrência de quedas, quando não provoca fraturas, pode ser esquecida pelos participantes: viés de memória. Além disso, pode ser considerado um fator de confundimento para a comparação o tipo de população analisada: foram observados moradores de um bairro da cidade de Campo Belo (Minas Gerais), cujos sujeitos supostamente não eram assistidos por nenhum programa vinculado à Universidade 29 , enquanto outros estudos aplicaram questionários em uma população de mulheres participantes de um programa de acompanhamento na Universidade Aberta da Terceira Idade ${ }^{12}$, em que havia atividades culturais e educacionais e cuidados médicos, e na Universidade do Estado do Rio de Janeiro ${ }^{30}$, onde praticavam atividades físicas. Esses sujeitos eram acompanhados por discentes/docentes da área de saúde, o que sugere maior atenção aos eventos que ocorrem em suas vidas. Já a incidência de fraturas na pesquisa de Chaimowicz et al. 29 foi maior do que a esperada ( $14,8 \%$ daqueles que caíram), confirmando que, quando elas ocorrem, há uma recordação mais intensa, em razão duma repercussão mais pronunciada nas suas vidas. A maioria dos sujeitos da pesquisa tinha entre 65 e 74 anos de idade, com visão $(71,4 \%)$ e audição (82\%) consideradas adequadas.

Coutinho \& Silva 31 observaram que a proporção de idosos que cai dentro de casa aumenta com o avançar da idade. Neste estudo, 46,7\% dos sujeitos que sofreram fratura relataram ter caído no último ano, enquanto $34,7 \%$ dos controles referiram apenas ter sofrido queda.

Parâmetros importantes a serem analisados como fatores de risco para fraturas, além do uso de medicamentos, foram identificados por Coutinho et al. 33 como índice de massa corporal abaixo de 20, diagnóstico de prejuízo cognitivo, ocorrência de acidente vascular cerebral e falta de controle do esfíncter uretral. Neste estudo, a maioria das fraturas ósseas ocorreu no fêmur (72\%) e braço/antebraço (19\%), e quase todos os casos incluídos na pesquisa necessitaram de algum tipo de procedimento cirúrgico. O local de ocorrência do evento em $67 \%$ dos sujeitos foi em casa. Já no estudo de Hamra et al. 32 essa porcentagem foi ainda maior: $74,6 \%$ dos acidentes 
ocorreram também em ambiente doméstico. Tal fato alerta para a necessidade de maior atenção dos idosos e cuidadores com os possiveis fatores de risco existentes no ambiente domicilar, como a presença de tapetes, a falta de corrimãos em escadas e banheiros, iluminação inadequada, excesso de móveis no ambiente, dentre outros 11 . Rozenfeld et al. 12 verificaram que a maioria das quedas $(78,2 \%)$ ocorreu fora de casa e teve como consequência problemas nos tecidos moles $(39,7 \%)$, feridas abertas $(23,9 \%)$, entorses $(11,1 \%)$, fraturas ósseas $(8,2 \%)$ e $26,7 \%$ dos sujeitos precisaram de atendimento ambulatorial após o acidente.

Na Tabela 2 podem ser observadas as associações nos estudos analisados, que foram realizadas de forma heterogênea, devido aos diferentes desenhos metodológicos: fato limitante para a realização de uma comparação estatística entre os estudos.

Guimarães \& Farinatti 30 encontraram que os indivíduos que consumiam 5 ou mais especialidades farmacêuticas apresentaram uma frequência percentual de quedas maior do que aqueles que consumiram menor quantidade; e as classes farmacêuticas aparentemente (termo utilizado pelo autor) mais associadas ao evento foram psicoativos e diuréticos. Segundo tal estudo, o uso de diuréticos pode causar hipotensão ortostática e arritmias, assim como depleção de volume e hipocalemia, fatores que podem levar à queda. Já na pesquisa de Coutinho \& Silva 31 , o uso de diuréticos reduz o risco de fraturas em $60 \%$. Tal fato pode ser explicado pelo seu efeito protetor, que diminui a ocorrência de fraturas por causa da redução urinária de cálcio, com consequente aumento da densidade óssea. Todavia, a ocorrência de queda em mulheres que utilizaram diuréticos na pesquisa de Rozenfeld et al. 12 e que apresentam também distúrbios musculoesqueléticos foi estatisticamente significante. Essa relação foi justificada neste estudo pelos seus efeitos iatrogênicos, como arritmias cardíacas, hipotensão postural, vertigem, fraqueza e distúrbios dos fluídos eletrolíticos.

A utilização de benzodiazepínicos e/ou psicoativos em geral apresentaram estatística significante com os eventos estudados em todos os estudos incluídos nesta revisão 12,29,30,31,32,33. O aumento do risco de quedas ou fraturas decorrentes de quedas entre usuários desses fármacos foi relacionado com a atividade sedativa e bloqueio $\alpha$-adrenérgico. Os hipnóticos e sedativos de meia vida longa podem provocar sedação residual importante, sobretudo em idosos, em função de mudanças em sua composição corporal tecidual. Há menor proporção de água corporal e maior de tecidos adiposos, podendo resultar em ocorrência de tonteiras, ataxia, confusão entre outros fatores predisponentes de quedas. Além disso, esses medicamentos possivelmente podem causar hipotensão, arritmias, sedação, tremores, relaxamento muscular ou fraqueza: caracteristicas diretamente relacionadas às quedas 12,29,30,31,32,33. Esses trabalhos estão de acordo com os achados da pesquisa do tipo de coorte de base populacional, que aponta que hipotensão ortostática, sedação, declínio cognitivo, osteoporose e fraqueza muscular estão altamente associados ao risco de quedas em idosos 41 , e também com estudos que apresentaram como sujeitos pacientes internados $42 \mathrm{e}$ ambulatoriais 43 .

Coutinho et al. 33 analisaram as fraturas decorrentes de quedas em pacientes internados em 5 hospitais do Rio de Janeiro, além da associação citada acima eles também notaram que os relaxantes musculares estavam associados a um risco aumentado de fraturas decorrentes de quedas. Isso pode se dever às reações como fraqueza, sonolência, sedação e efeitos anticolinérgicos predisponentes para a ocorrência desse evento.

Dentre os medicamentos associados a fraturas decorrentes de quedas, Coutinho \& Silva 31 também encontraram relação com o uso de bloqueadores de canais de cálcio, que foi atribuída ao efeito inotrópico negativo provocado por esses fármacos, que normalmente é compensado mediante estimulação adrenérgica cardíaca para equilibrar a pressão arterial. Esse reflexo muitas vezes ocorre mais lentamente nos idosos favorecendo a ocorrência de quedas. Assim, o uso de bloqueadores de canais de cálcio são potenciais causadores de queda, em razão da reação de hipotensão ortostática, porém, ao contrário do estudo anterior, este 33 referiu um possível efeito protetor para o risco de fraturas decorrentes de quedas. Segundo os autores, esse achado pode ser devido ao confundimento residual de autorrelato (termo usado pelo autor) decorrente do estado de saúde dos participantes 33 .

O estudo de Hamra et al. 32 foi do tipo casocontrole, sendo os casos pacientes internados pelo Serviço de Ortopedia e Traumatologia, vítimas de fraturas decorrentes de quedas. As fraturas no fêmur ocorreram em 53,1\% dos indivíduos, enquanto as no punho ocorreram em 26,3\%. A associação do uso de medicamentos e a ocorrência de fraturas foi realizada pela análise estatística de OR. Foi notado que o número de fraturas decorrentes de quedas entre pacientes usuários de captopril, clonazepam, hidroclorotiazida, cinarizina e flunarizina foi bem maior no grupo de casos do que no de controle, fato que se deve provavelmente aos efeitos adversos desses fármacos que levam a quedas, como hipotensão 
postural, sonolência, tonturas, aumento na frequência de micção.

Diante desses estudos, pode-se verificar que as classes de medicamentos psicoativos ou psicotrópicos, que abrangem os medicamentos antidepressivos, antipsicóticos, ansiolíticos, hipnóticos e sedativos, foram consideradas potencialmente associadas ao risco de queda ou de fraturas decorrentes de quedas, por estarem associadas ao evento em todos os estudos incluídos nesta revisão sistemática, embora a terminologia variasse (classe farmacológica ou medicamento). Este resultado está de acordo com o exposto por meta-análises ou revisões realizadas em outros países 8,44,45,46, além do clássico estudo de Beers 20,21.

Seria indicado calcular uma medida única de associação estatística, entretanto não foi possível fazê-la por conta da dificuldade de correlacionálas e também pela diversidade de classificação dos medicamentos descritos. A grande variação de medidas de associação utilizada pelos estudos inviabilizou a construção de gráfico ou o cálculo de uma medida-sumário que englobasse todos os resultados. Para isso seria necessário calcular uma medida que não foi descrita em alguns estudos por meio dos dados apresentados, e caso se fizesse isso, poder-se-ia incorrer em erro, uma vez que nos artigos não estão demonstrados os dados de forma precisa para a obtenção de tal cálculo. Quatro dos artigos estudados 12,31,32,33 apresentam a OR, (embora Hamra et al. 32 a apresentem em porcentagem); dentre os outros, um deles 30 é possível calcular a OR com base nos dados citados, mas o um outro 29 não apresenta dados suficientes para tal. Mesmo neste que seja possível calcular a OR, ela seria calculada valendo-se de todos os medicamentos sem definição de classe medicamentosa específica.

As medidas de associação que devem ser utilizadas nos estudos seccionais são razão de prevalência e diferença de prevalência. A OR pode ser calculada em qualquer desenho de estudo e deve ser a medida de associação usada nos estudos caso-controle 47 . No estudo de Guimarães \& Farinatti 30 , a medida de associação utilizada foi a frequência relativa, que apresentou a razão entre o total de quedas no ano anterior e o total de indivíduos expostos a um tipo de medicação específica, mas não foi demonstrada a tabela de contingência para os expostos e não expostos aos medicamentos e os que sofreram queda ou não; dessa forma, a associação relatada pelo estudo foi considerada questionável. A forma de associar os medicamentos com as quedas realizada por Hamra et al. 32 também foi inconclusiva, dado que calculou uma OR para todos os medicamentos em conjunto, não obtendo resultado consisten- te. A associação de medicação alvo com quedas foi realizada de forma pouco clara. No presente estudo, comparou-se a frequência de uso de cada medicação pelos idosos do grupo de fraturas e do controle, porém tal relação se tornou incompreensível quando se observou que outros medicamentos - que não foram associados como fatores de risco para fraturas - apresentaram resultados semelhantes.

Os resultados dos estudos incluídos nesta revisão endossam que as fraturas são uma das consequências mais comuns decorrentes de quedas, sendo as mais frequentes a do fêmur $(62 \%)$, seguidas pelas de rádio (12,5\%) e clavícula $(6,25 \%) 48$. No estudo de Siqueira et al. 13, dentre os idosos que caíram no ano anterior da pesquisa, 12,1\% deles tiveram fraturas decorrentes de quedas, sendo $46 \%$ delas nos membros superiores, $28 \%$ nos membros inferiores, $11 \%$ no tronco, $5,5 \%$ na face e o restante nas demais partes do corpo. Em uma revisão sistemática realizada na Espanha 49, observou-se que as principais consequências relacionadas às quedas foram os índices de fratura, que variaram de $1,2 \%$ a $16,5 \%$, cujos valores estão de acordo com os dados anteriormente mencionados no Brasil.

Um dos principais achados desta revisão sistemática é a necessidade de novas pesquisas para investigar o uso de medicamentos como fator de risco para quedas em idosos. Para tanto é sugerido que sejam conduzidos estudos epidemiológicos observacionais com rigorosa metodologia e análise estatística adequada dos dados, para que os resultados desses possam ser aplicados na prática geriátrica.

A falta de estudos que analisem diretamente o uso de medicamentos como fator predisponente de quedas em idosos gera problemas de saúde pública como escolha inadequada de medicamentos e exposições indevidas ao risco de quedas que podem ser fatais ou apresentarem desfechos graves como: iatrogenias, deterioração funcional, uso de medicamentos para suprir a dor, utilização de serviços de saúde, hospitalizações e mortalidade. Além disso, na maioria das vezes, ocorrem sequelas psicológicas como medo de cair com consequente limitação das atividades e da mobilidade, diminuição da atividade física, isolamento social e depressão. Apesar de a associação esboçada já estar consolidada em vários estudos estrangeiros, é necessário que sejam feitas novas pesquisas nessa área, particularmente no Brasil, para verificar a existência de prescrições de medicamentos potencialmente associados às quedas e a ocorrência desses eventos em idosos brasileiros, contribuindo para um uso racional de medicamentos em geriatria. 


\section{Limitações da revisão}

A falta de homogeneidade entre os estudos levou a uma limitação na análise. A dificuldade encontrada nas pesquisas analisadas foi referente às diferentes formas de referenciar as classes medicamentosas nos artigos, o que poderia conduzir a uma análise tendenciosa e inconclusiva. Alguns trabalhos classificam todos os medicamentos psicoativos em conjunto, independentemente da classe farmacológica específica, enquanto outros os citam em grupos e subgrupos de medicamentos. Tal diferença de classificação dificulta a análise e a comparação entre os artigos, pois a diferença entre o uso de medicamentos, quer seja com indicação ansiolítica, sedativa ou como hipnótica, muitas vezes pode ser por conta de ajustes de doses e de meia vida de eliminação. Um exemplo especial é o caso dos benzodiazepínicos, que podem ser prescritos para todas as finalidades acima apontadas. É possível que haja comprometimento de análise e comparação entre os estudos selecionados, até em termos de recomendação terapêutica e alerta de perigo para uso na população referida, pois se pode considerar equivocadamente que somente em altas doses esses medicamentos provocam reações adversas. É importante que as pesquisas científicas frisem a indicação dos medicamentos e doses utilizadas para adoção como medida cautelar na assistência ao paciente geriátrico.

A própria metodologia adotada nesta revisão, que limitou o idioma utilizado, a escolha dos descritores e a busca em bases de dados específicas, necessárias e esperadas para sistematizar o tra- balho, pode ter promovido a perda de pesquisas relevantes por estarem em bases de dados que não foram utilizadas ou que apresentaram descritores diferentes dos que foram empregados no estudo, assim como por terem sido publicadas em idiomas diferentes dos aqui selecionados.

\section{Conclusões}

Os estudos realizados no Brasil são relativamente recentes (15 anos) e enfocam as quedas entre idosos associadas a fatores fisiopatológicos próprios do envelhecimento, sem relacionar o uso de medicamentos em geriatria.

A natureza desses estudos (observação de reação adversa após o uso de medicamentos) justifica o seu desenho epidemiológico retrospectivo, em se tratando da questão ética, mesmo se sabendo das limitações e desvantagens de estudos retrospectivos e seus vieses de informação e memória.

Esta revisão demonstrou que o uso de alguns medicamentos por idosos, especialmente diuréticos e benzodiazepínicos, representa um potencial fator de risco de quedas. Essa abordagem pode contribuir na farmacoterapia geriátrica racional, sobretudo para aqueles pacientes que sofrem de doenças crônicas e que necessitam de utilizar tais classes medicamentosas. Estudos prospectivos e de intervenção que examinem os efeitos dos fármacos na qualidade de vida dos pacientes são indicados. 


\section{Resumo}

Quedas em idosos estão frequentemente relacionadas ao uso de medicamentos e geralmente levam a um prognóstico ruim, representando um problema de saúde pública. O objetivo deste trabalho foi identificar estudos publicados no Brasil, examinando o uso de medicamentos como fator de risco para quedas ou fraturas decorrentes das quedas em idosos. As bases de dados utilizadas foram LILACS, PubMed, SciELO, utilizando os descritores falls, elderly, medication/pharmaceutical preparations/medicines/drugs ou ainda specific groups of medications. Foram identificados 340 artigos; dentre eles, 53 cumpriram com os critérios de inclusão, porém apenas 6 estudos farmacoepidemiológicos de quedas por idosos foram realizados no Brasil. Os principais grupos farmacológicos associados ao aumento do risco de queda foram: antidepressivos, sedativos, ansiolíticos, diuréticos. Tendo em vista o envelhecimento da população no Brasil, necessário é que sejam realizadas e incentivadas pesquisas bem delineadas com tal população para produzir informação científica idônea e promover uso racional de medicamentos em geriatria.

Acidentes por Quedas; Idoso; Uso de Medicamentos

\section{Colaboradores}

C. P. Rezende contribuiu com a concepção e projeto, análise e interpretação dos dados, redação do artigo, revisão crítica relevante do conteúdo intelectual e aprovação final da versão a ser publicada. M. R. G. GaedeCarrillo contribuiu com a redação do artigo, revisão crítica relevante do conteúdo intelectual e aprovação final da versão a ser publicada. E. C. O. Sebastião contribuiu com a concepção e projeto, análise e interpretação dos dados, redação do artigo, revisão crítica relevante do conteúdo intelectual e aprovação final da versão a ser publicada.

\section{Agradecimentos}

Agradecemos ao Núcleo de Estudos sobre o Envelhecimento (NEsE) da Universidade Federal de Ouro Preto pelo apoio.

\section{Referências}

1. Lima-Costa MF, Veras R. Saúde pública e envelhecimento. Cad Saúde Pública 2003; 19:700-1.

2. Siqueira RL, Botelho MIV, Coelho FMG. A velhice: algumas considerações teóricas e conceituais. Ciênc Saúde Coletiva 2002; 7:899-906.

3. Organização Mundial da Saúde. Envelhecimento ativo: uma política de saúde. Brasília: Organização Pan-Americana da Saúde; 2005.

4. Ministério da Saúde. Estatuto do idoso. Brasília: Ministério da Saúde; 2003.

5. Chaimowicz F. A saúde dos idosos brasileiros às vésperas do século XXI: problemas, projeções e alternativas. Rev Saúde Pública 1997; 31:184-200.

6. Rodrigues MAP, Facchini LA, Piccini RX, Tomasi E, Thumé E, Silveira DS, et al. Uso de serviços básicos de saúde por idosos portadores de condições crônicas. Rev Saúde Pública 2009; 43:604-12.
7. Loyola Filho AI, Uchoa E, Firmo JOA, Lima-Costa MF. Estudo de base populacional sobre o consumo de medicamentos entre idosos: Projeto Bambuí. Cad Saúde Pública 2005; 21:545-53.

8. Hartikainen S, Lonnroos E, Louhivuori K. Medication as a risk factor for falls: critical systematic review. J Gerontol 2007; 62A:1172-81.

9. Menezes RL, Bachion MM. Estudo da presença de fatores de riscos intrínsecos para quedas, em idosos institucionalizados. Ciênc Saúde Coletiva 2008; 13:1209-18.

10. Freitas EV, Py L. Tratado de geriatria e gerontologia. 2a Ed. Rio de Janeiro: Editora Guanabara Koogan; 2006 
11. Pereira SRM, Buksman S, Perracini M, Py L, Barreto KML, Leite VMM. Quedas em idosos. In: Jatene FB, Cutait R, Eluf Neto J, Nobre MR, Bernardo WM, organizadores. Projeto diretrizes. v. 1. São Paulo: Associação Médica Brasileira/Brasília: Conselho Federal de Medicina; 2002. p. 405-14.

12. Rozenfeld S, Camacho LA, Veras RP. Medication as a risk factor for falls in older women in Brazil. Rev Panam Salud Pública 2003; 13:369-75.

13. Siqueira FV, Facchini LA, Piccini RX, Tomasi E, Thumé E, Silveira DS, et al. Prevalência de quedas em idosos e fatores associados. Rev Saúde Pública 2007; 41:749-56.

14. Rubenstein LZ. Falls in older people: epidemiology, risk factors and strategies for prevention. Age Ageing 2006; 35 Suppl 2:ii37-41.

15. Ribeiro AP, Souza ERS, Atie S, Souza AC, Schilithz AO. A influência das quedas na qualidade de vida de idosos. Ciênc Saúde Coletiva 2008; 13:1265-73.

16. Petridou ET, Dikalioti SK, Dessypris N, Skalkidis I, Barbone F, Fitzpatrick P, et al. The evolution of unintentional injury mortality among elderly in Europe. J Aging Health 2008; 20:159-82.

17. Aschkenasy MT, Rothenhaus TC. Trauma and falls in the elderly. Emerg Med Clin North Am 2006; 24:413-32.

18. Gawryszewski VP. A importância das quedas no mesmo nível entre idosos no estado de São Paulo. Rev Assoc Med Bras 2010; 56:162-7.

19. Perracini M. Desafios da prevenção e do manejo de quedas em idosos. BIS Bol Inst Saúde (Impr) 2009;47:45-8.

20. Beers MH, Ouslander JG, Rollingher I, Reuben DB, Brooks J, Beck JC. Explicit criteria for determining inappropriate medication use in nursing home residents. UCLA Division of Geriatric Medicine. Arch Intern Med 1991; 151:1825-32.

21. Beers MH. Explicit criteria for determining potentially inappropriate medication use by the elderly. An update. Arch Intern Med 1997; 157:1531-6.

22. Fick DM, Cooper JW, Wade WE, Waller JL, Maclean JR, Beers MH. Updating the Beers criteria for potentially inappropriate medication use in older adults: results of a US consensus panel of experts. Arch Intern Med 2003; 163:2716-24.

23. Gorzoni ML, Fabbri RMA, Pires SL. Critérios de Beers-Fick e medicamentos genéricos no Brasil. Rev Assoc Med Bras 2008; 54:353-6.

24. Ribeiro AQ, Araújo CMC, Acúrcio FA, Magalhães SMS, Chaimowicz F. Qualidade do uso de medicamentos por idosos: uma revisão dos métodos de avaliação disponíveis. Ciênc Saúde Coletiva 2005; 10:1037-45.

25. Coelho Filho JM, Marcopito LF, Castelo A. Perfil de utilização de medicamentos por idosos em área urbana do Nordeste do Brasil. Rev Saúde Pública 2004; 38:557-64.

26. Santos RM, Sette IMF, Bélem LF. Drug use by elderly inpatients of a philanthropic hospital. Braz J Pharm Sci 2011; 47:391-8.

27. Bennett S, Bennett JW. The process of evidencebased practice in occupational therapy: informing clinical decisions. Aust Occup Ther J 2000; 47: 171-80.
28. Nordic Council on Medicines. Lineamentos para classificación ATC y la asignación de DDD. Oslo: WHO Collaborating Centre for Drug Statistics Methodology; 2001.

29. Chaimowicz F, Ferreira TJXM, Miguel DFA. Use of psychoactive drugs and related falls among older people living in a community in Brazil. Rev Saúde Pública 2000; 34:631-5.

30. Coutinho ESF, Silva SD. Medication as a risk factor for falls resulting in severe fractures in the elderly. Cad Saúde Pública 2002; 18:1359-66.

31. Guimarães JMN, Farinatti PTV. Análise descritiva de variáveis teoricamente associadas ao risco de quedas em mulheres idosas. Rev Bras Med Esporte 2005; 11:299-305.

32. Hamra A, Ribeiro MB, Miguel OF. Correlação entre fratura por queda em idosos e uso prévio de medicamentos. Acta Ortop Bras 2007; 15:143-5.

33. Coutinho ESF, Fletcher A, Bloch KV, Rodrigues LC. Risk factors for falls with severe fracture in elderly people living in a middle-income country: a case control study. BMC Geriatrics 2008; 8:1-7.

34. Veras R. Envelhecimento populacional contemporâneo: demanda, desafios e inovações. Rev Saúde Pública 2009; 43:548-54.

35. Riera R, Trevisani VFM, Ribeiro JPN. Osteoporose - a importância da prevenção de quedas. Rev Bras Reumatol 2003; 43:364-8.

36. Gai J, Gomes L, Nóbrega OT, Rodrigues MP. Fatores associados a quedas em mulheres idosos residentes na comunidade. Rev Assoc Med Bras 2010; 56:327-32.

37. Pereira SEM, Mendonça LMC. Osteoporose e osteomalacia. In: Freitas EV, Py L, organizadores. Tratado de geriatria e gerontologia. 2a Ed. Rio de Janeiro: Editora Guanabara Koogan; 2006. p. 799-805.

38. Siqueira FV, Facchini LA, Silveira DS, Piccini RX, Tomasi E, Thumé E, et al. Prevalence of falls in elderly in Brazil: a countrywide analysis. Cad Saúde Pública 2011; 27:1819-26.

39. Oliveira PP, Marinheiro LPF, Wender MCO, Roisenberg F, Lacativa PGS. Prevalência de fraturas vertebrais e fatores de risco em mulheres com mais de 60 anos de idade na cidade de Chapecó, Santa Catarina, Brasil. Cad Saúde Pública 2010; 26: 1777-87.

40. Wilson NM, Hilmer SN, March LM, Cameron ID, Lord SR, Seibel MJ, et al. Associations between drug burden index and falls in older people in residential aged care. J Am Geriatr Soc 2011; 59:87580.

41. Kragh A, Elmsta S, Atroshi I. Older adults' medication use 6 months before and after hip fracture: a population-based cohort study. J Am Geriatr Soc 2011; 59:863-8.

42. Chang CM, Chen MJ, Tsai C, Ho L, Hsieh H, Chau $\mathrm{Y}$, et al. Medical conditions and medications as risk factors of falls in the inpatient older people: a case-control study. Int J Geriatr Psychiatry 2011; 26:602-7.

43. Kojima T, Akishita M, Nakamura T, Nomura K, Ogawa S, Iijima K, et al. Association of polypharmacy with fall risk among geriatric outpatients. Geriatr Gerontol Int 2011; 11:438-44. 
44. Boyle N, Naganathan V, Cumming RG. Medication and falls: risk and optimization. Clin Geriatric Med 2010; 4:585-605.

45. Leipzig RM, Cumming RG, Tinetti ME. Drugs and falls in older people: systematic review and metaanalyses: I. Psychotropic drugs. J Am Geriatr Soc $1999 ; 47: 30-9$

46. Woolcott JC, Richardson KJ, Wiens MO, Patel B, Marin J, Khan KM, et al. Meta-analysis of the impact of 9 medication classes on falls in elderly persons. Arch Intern Med 2009; 169:1952-60.

47. Kale PL, Costa AJL, Luiz RR. Medidas de associação e medidas de impacto. In: Medronho RA, Bloch KV, Luiz RR, Werneck GL, organizadores. Epidemiologia. 2a Ed. Rio de Janeiro: Editora Atheneu; 2009. p. 181-90.
48. Fabrício SCC, Rodrigues RAP, Costa Júnior ML. Falls among older adults seen at a São Paulo State public hospital: causes and consequences. Rev Saúde Pública 2004; 38:93-9.

49. Gama ZAS, Conesa AG, Ferreira MS. Epidemiología de caídas de ancianos en España. Una revisión sistemática, 2007. Rev Esp Salud Pública 2008; 82: 43-56.

Recebido em 26/Abr/2012

Versão final reapresentada em 12/Jul/2012

Aprovado em 17/Ago/2012 